Polymer Journal, Vol. 6, No. 1, p 1-6 (1974)

\title{
ESR Studies on Primary Processes in Radiation-Induced Reactions. V. Polymerization of 1,3-Butadiene on Silica Gel at $77^{\circ} \mathrm{K}$ Studied by Computer Analysis
}

\author{
Tetsuo Shiga and Seizo OKamura \\ Department of Polymer Chemistry, Kyoto University, \\ Kyoto, 606, Japan.
}

(Received January 4, 1973)

\begin{abstract}
The primary processes of radiation-induced polymerization of 1,3butadiene-1,1,4,4- $d_{4}$ on silica gel have been investigated quantitatively by means of ESR. The spectra containing several superimposed components have been analysed by a computer. The cation radical of butadiene is formed by the interaction with irradiated silica gel. Polymerization is initiated by the butadiene cation radicals followed by the addition of butadiene molecules and terminated by the recombination between the propagating allyl-type cations and electrons. The rate constant of the first step of polymerization, i.e., the addition of the butadiene molecule to the butadiene cation radical is discussed.
\end{abstract}

KEY WORDS ESR / Computer / Radiation-Induced Polymerization / Adsorbed State / Silica Gel / Rate Constant / 1,3-Butadiene / Cation Radical /

In the previous work the species from 1,3butadiene adsorbed on silica gel have been investigated by means of ESR following $\gamma$ irradiation. ${ }^{1}$ The thermally unstable ESR signal observed at low concentration of butadiene $(0.1$ mol\%) on silica gel has been assigned to the cation radical of butadiene. The identification of the butadiene cation radical has been supported by using 1,3-butadiene-1,1,4,4- $d_{4}$ and by the effect of a cation scavenger. ${ }^{2}$ It has been suggested that the butadiene cation radical reacts with a butadiene molecule, i.e., initiates radiation-induced cationic polymerization to form the allyl-type radical on another end of a propagating chain. ${ }^{1}$

In the present investigation the reaction mechanisms are studied quantitatively by using a computer program in order to analyse ESR spectra containing several superimposed components and also by studying kinetics for the primary processes in radiation-induced polymerization.

\section{EXPERIMENTAL}

The silica gel was dried at $650^{\circ} \mathrm{C}$ for $24 \mathrm{hr}$ by a least-squares fit procedure using a number except for the case where the effect of the drying temperatures of the silica gel on the yield of species was investigated. The procedures for purifying and drying 1,3-butadiene, for sample preparation and for irradiation were mentioned previously. ${ }^{1}$ The ESR spectra were measured in the dark with a Varian E-9 X-band spectrometer employing $100-\mathrm{kHz}$ magnetic modulation at a microwave power of $0.06 \mathrm{~mW}$.

The ESR spectrum obtained experimentally, which is composed of several species, was analysed by a computer program ${ }^{3}$ which can determine the relative concentration of each species. Spectra intended for computer analysis were recorded in digital form on paper tape by means of C-1024 time-averaging computer supplemented with a TMC Model 220 data output unit and a Tally Model 420 tape perforator connected to the spectrometer.

The analysis of spectra containing several superimposed components was fitted to an equation of the type

$$
F\left(x_{i}\right)=\sum_{j=1}^{m} c_{j} f_{j}\left(x_{i}\right)
$$


of equidistant field points $x_{i}$. Here $c_{j}$ represents the fraction of the component $f_{j}$ present. These components are simulated in the program ${ }^{3}$ which employs the appropriate parameters for the coupling constants $a$, derivative linewidths $\Delta H_{p p}$, and $g$ factors. Either Lorentzian or Gaussian derivative line-profiles can be used. The components $f_{j}$ can also be read in digital form on paper tape, available from the experiment. Output is the fractions $c_{j}$ with errors and concentrations $c_{j} f_{j}(x)$, together with plots of the composed spectrum, its components spectra, and the spectrum obtained by the least-square fit. In addition, one or more of the components can be subtracted allowing the line profile of weak absorptions hidden under the main spectrum to be estimated.

The performance of this program is shown in Figure 1. The experimental spectrum $F$ is assumed to be composed of four components of $f_{j}(m=4)$. The spectra $f_{1}$ and $f_{2}$ were read as numerical line shapes on paper tapes, while the spectra $f_{3}$ and $f_{4}$ were simulated in the program. The spectrum $B$ is the spectrum obtained by the least-square fit. The fraction of each component, $F_{j}=c_{j} f_{j} / F$, is $F_{1}=0.25, \quad F_{2}=0.28, \quad F_{3}=$ 0.19 , and $F_{4}=0.28$. Relative errors of the fraction $c_{j}$ in this case are $1.4 \%$ for $c_{1}, 3.8 \%$ for $c_{2}, 1.9 \%$ for, $c_{3}$ and $4.5 \%$ for $c_{4}$.

\section{RESULTS}

Three kinds of radicals have been produced from 1,3-butadiene by $\gamma$-irradiation. ${ }^{1}$ Corresponding radicals from 1,3-butadiene-1,1,4,4- $d_{4}$ are $\mathrm{CD}_{2}=\mathrm{CH}-\dot{\mathrm{C}} \mathrm{H}-\mathrm{CD}_{2}-, \quad\left(\mathrm{CD}_{2}-\mathrm{CH}=\mathrm{CH}-\right.$ $\left.\mathrm{CD}_{2}\right)^{+} \cdot$, and $\mathrm{CD}_{2} \mathrm{H}-\dot{\mathrm{CH}}-\mathrm{CH}=\mathrm{CD}_{2}$ which are the allyl-type radical located at the end of polymer chain, the butadiene cation radical, and hydrogen atom addition-type radical respectively.

The spectrum $F$ in Figure 1 is the experimental one recorded in the dark from the sample containing $0.13 \mathrm{~mol} \%$ of butadiene- $d_{4}$. The spectrum $B$ is the computed one from the signal from silica gel $\left(f_{1}\right)^{4}, \mathrm{CD}_{2}=\mathrm{CH}-\dot{\mathrm{C}} \mathrm{H}-\mathrm{CD}_{2}-\left(f_{2}\right)$, $\left(\mathrm{CD}_{2}-\mathrm{CH}=\mathrm{CH}-\mathrm{CD}_{2}\right)^{+} \cdot\left(f_{3}\right)$ and $\mathrm{CD}_{2} \mathrm{H}-\dot{\mathrm{C}} \mathrm{H}-$ $\mathrm{CH}=\mathrm{CD}_{2}\left(f_{4}\right)$.

Agreement between the spectrum $F$ and $B$ is
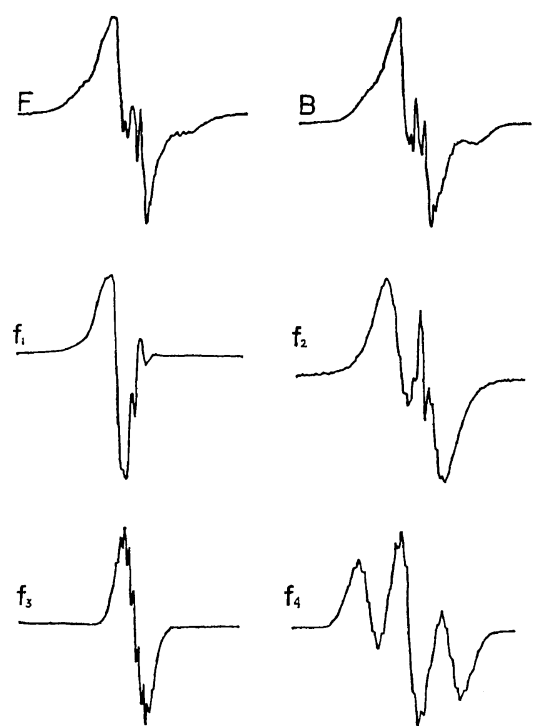

Figure 1. The analysis of ESR spectrum containing several superimposed components: F, experimental; $f_{1}, f_{2}$, components 1 and 2 read from a paper tape; $f_{3}$, component 3 simulated by parameters of $a_{1}=$ $3.16 \mathrm{G}(2 \mathrm{H}), a_{2}=1.74 \mathrm{G}(4 \mathrm{D})$ and a Lorentzian $\Delta H_{p p}=1.7 \mathrm{G} ; f_{4}$, component 4 simulated by parameters of $a_{1}=13.8 \mathrm{G}(1 \mathrm{H}), a_{2}=16.4 \mathrm{G}(1 \mathrm{H}), a_{3}=$ $3.9 \mathrm{G}(1 \mathrm{H}), a_{4}=2.5 \mathrm{G}(2 \mathrm{D}), a_{5}=2.1 \mathrm{G}(1 \mathrm{D}), a_{6}=$ $2.3 \mathrm{G}(1 \mathrm{D})$ and a Lorentzian $\Delta H_{p p}=2.4 \mathrm{G}$; $\mathrm{B}$, spectrum obtained from the least square fit with relative concentration of $f_{1}: f_{2}: f_{3}: f_{4}=0.25: 0.28$ : $0.19: 0.28$.

very good. The yields of $f_{1}, f_{2}, f_{3}$, and $f_{4}$ are studied for several concentrations of butadiene$d_{4}$ on silica gel. The ESR spectra from samples with different butadiene contents (0.13-5.8 mol\%) are shown in Figure 2. They clearly show that the hole centers $\left(\mathbf{S}^{+} \cdot\right)$ trapped on the surface of silica gel at $g=2.0070^{4}$ and the butadiene cation radical $\left(\mathbf{B}^{+} \cdot\right)$ decrease with increasing butadiene content, while the radical $\mathrm{RI}, \quad \mathrm{CD}_{2}=\mathrm{CH}-\dot{\mathrm{C}} \mathrm{H}-\mathrm{CD}_{2}-, \quad(\dot{\mathrm{B}}-)$, increases with butadiene content. The concentrations of these species are determined by using a computer program in which the concentration of the silica gel hole at $g=2.0070$ is taken to be $c_{1} f_{1}$ in Figure 1, because the contribution of the hole signal to the spectrum $f_{1}$ is predominant. The yields of $f_{1}, f_{2}, f_{3}$, and $f_{4}$ at different butadiene contents are shown in Figure 3 at the total dose of $2.8 \times 10^{5} \mathrm{rad}$. The silica gel hole decays 


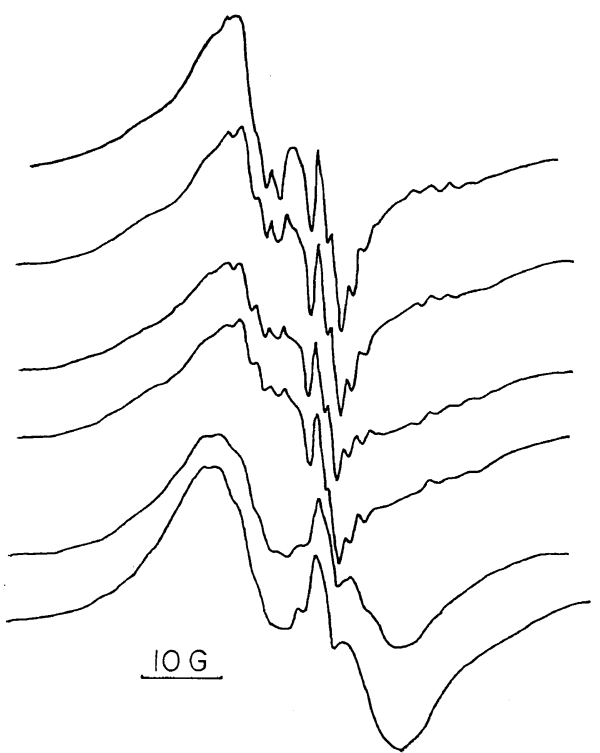

Figure 2. ESR spectra from samples with different butadiene contents; the content is $0.13,0.25,0.60$, 3.0 , and $5.8 \mathrm{~mol} \%$ reading from top to bottom.

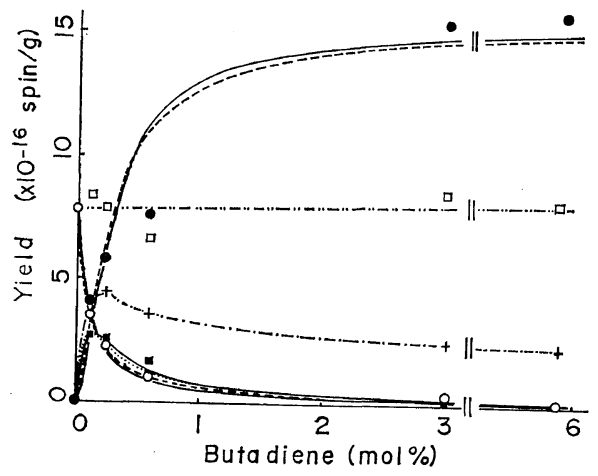

Figure 3. The effect of the butadiene content on the yields at $77^{\circ} \mathrm{K}$ of the silica gel hole, $\mathrm{S}^{+}$, the butadiene cation radical, $\mathrm{B}^{+}$, the radical $\mathrm{RI}$ and the radical RII: $\bigcirc, \mathrm{S}^{+} ; \mathbf{\square}, \mathrm{B}^{+}$; $\bullet$, RI; +, RII; $\square, \mathrm{S}^{+}+\mathrm{B}^{+}+\mathrm{RI} / 2$; $\longrightarrow$, the theoretical curves for the Model I in the text; -..-., the theoretical curves for the Model II in the text.

continuously. The butadiene cation radical goes through a maximum and then decays. The radical $\mathrm{RI}$ increases and reaches a plateau. The radical $\mathrm{RII}, \mathrm{CD}_{2} \mathrm{H}-\dot{\mathrm{C}} \mathrm{H}-\mathrm{CH}=\mathrm{CD}_{2}$, decreases only slightly with increasing butadiene cation radical and one half of the radical RI is almost constant with respect to the butadiene content

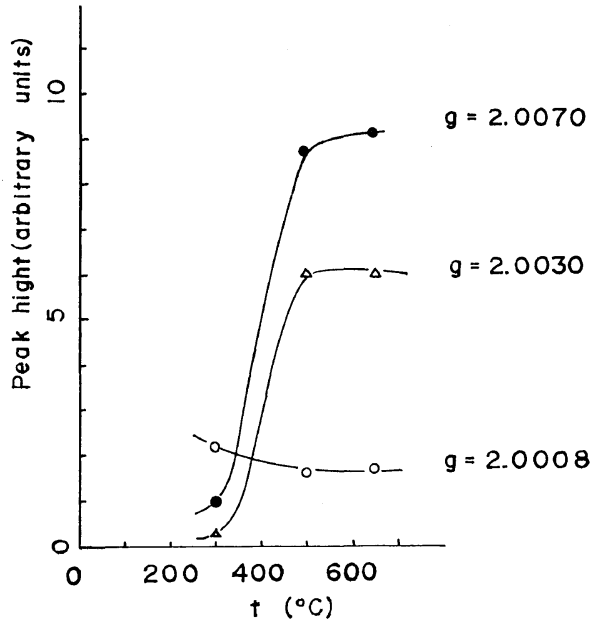

Figure 4. The effect of drying temperature of sillica gel on the yield of the paramagnetic centers in irradiated silica gel.

and is equal to the yield of the silica gel hole in the absence of butadiene as shown in Figure 3.

The yields of the hole at $g=2.0070$ and electrons at $g=2.0030$ and at $g=2.0008$ in irradiated silica gel in the absence of adsorbates are shown in Figure 4 for silica gel dried at different temperatures. The height in Figure 4 was taken from the peak intensity in a spectrum. Thus, the plot only shows the effect of drying temperatures on the yields of these species. Holes at $g=2.0070$ and electrons at $g=2.0030$ increase with increasing drying temperatures and reach a plateau at temperatures higher than $500^{\circ} \mathrm{C}$, while the electrons at $g=2.0008$ decrease

Table I. Absorption amplitudes of the different components in silica gel treated in various ways before irradiation. The samples were degassed at $500^{\circ} \mathrm{C}$ and irradiated to a dose of 1.5 Mrad. Identification of each center was made previously ${ }^{4}$

\begin{tabular}{lrlll}
\hline $\begin{array}{l}\text { Sample } \\
\text { treatment }\end{array}$ & \multicolumn{4}{c}{ Absorption amplitude } \\
\cline { 2 - 5 } & $g=2.0008$ & $g=2.0030$ & $g=2.0070$ & $g=2.011$ \\
\hline None & 1.0 & 1.0 & 1.0 & 1.0 \\
Boiled with $\mathrm{HCl}$ & 2.4 & 0.1 & 0.21 & 0.26 \\
Boiled with $\mathrm{HNO}_{3}$ & 1.2 & 1.3 & 0.93 & 0.74
\end{tabular}




\section{T. Shiga and S. OKamura}

slightly with these temperatures. The electrons at $g=2.0030$ show similar behavior to the hole at $g=2.0070$. The effects of the treatment of the silica gel before irradiation also indicates that the electrons at $g=2.0030$ run parallel to the holes, but the electrons at $g=2.0008$ do not (Table I).

Neither the butadiene cation radicals nor the holes at $g=2.0070$ nor the electrons at $g=2.0030$ are produced from the sample in which silica gel is dried at a temperature of less than $250^{\circ} \mathrm{C}$. The yield of the butadiene cation radical shows a similar behavior to that of the hole with respect to drying temperatures of silica gel between 300 and $650^{\circ} \mathrm{C}$.

\section{DISCUSSION}

The spectrum $f_{2}$ in Figure 1 is a doublet spectrum superimposed with the signal from electrons at $g=2.0008$. The doublet spectrum changes reversibly into a better resolved one at $200^{\circ} \mathrm{K}$ as is also observed in the radical RI of the ordinary butadiene. ${ }^{1}$ The simulation for the spectrum of the radical RI of butadiene- $d_{4}$ at $200^{\circ} \mathrm{K}$ gives assignments of proton coupling constants of 14.9 and $13.8 \mathrm{G}$ to two $\alpha$ protons attached to carbon $1,13.8 \mathrm{G}$ to a proton attached to carbon 3 and $12.8 \mathrm{G}$ to two equivalent $\alpha$ protons attached to carbon 4 for the radical RI of the ordinary butadiene.

The inhibiting effect of a cation scavenger on the formation of the silica gel hole and the butadiene cation radical ${ }^{2}$ and the similar behavior in the formation of the silica gel holes and the butadiene cation radicals with respect to the drying temperatures of the silica gel clearly indicate that the silica gel hole is closely related to the formation of the butadiene cation radical. From the behavior of $\mathbf{B}^{+}$. and $\mathbf{S}^{+}$. in Figure 3 two reaction mechanisms for the formation of the butadiene cation radical are considered. One is that the butadiene cation radical is produced by a positive charge transfer from the silica gel hole which is produced by the ionizing radiation, (1) and (2) in Model I. Another is that the silica gel hole and the butadiene cation radical are produced in competing reactions with certain active centers created during the irradiation, (6), (7), and (8) in Model II. The constancy: $\left[\mathbf{S}^{+} \cdot\right]+\left[\mathbf{B}^{+} \cdot\right]+[\dot{\mathbf{B}}-] / 2$ which is equal to $\left[\mathbf{S}^{+} \cdot\right]_{0}$, the yield in the absence of butadiene, and only a slight decrease of [RII] with increasing butadiene content indicate that the radical RII is not necessarily involved in the reaction mechanisms of $\mathrm{S}^{+}$. and $\mathrm{B}^{+}$, , and also that the radical RI is located at both ends of a polymer chain. Reaction mechanisms considered are as follows:

$$
\begin{aligned}
& \text { Model I } \\
& \mathbf{S}_{m \rightarrow} \mathbf{S}^{+} \cdot+\mathrm{e}^{-} \\
& \mathrm{S}^{+} \cdot+\mathrm{B} \rightarrow \mathrm{S}+\mathrm{B}^{+} . \quad k_{1}{ }^{\prime} \text {. } \\
& \mathrm{B}^{+} \cdot+\mathrm{B} \rightarrow \dot{\mathrm{B}}-\mathrm{B}^{+} \quad k_{2}{ }^{\prime} \\
& \dot{\mathrm{B}}-\mathrm{B}^{+}+\mathrm{B} \rightarrow \dot{\mathrm{B}}-\mathrm{B}-\mathrm{B}^{+} \\
& \text {…....... } \\
& \dot{\mathbf{B}}-(\mathbf{B})_{n}-\mathbf{B}^{+}+\mathrm{e}^{-} \cdots \dot{\mathbf{B}}-(\mathbf{B})_{n}-\dot{\mathbf{B}}
\end{aligned}
$$

Model II

$$
\begin{array}{ll}
\mathrm{S} m \mathrm{~S}^{*} & \\
\mathrm{~S}^{*} \rightarrow \mathrm{S}^{+} \cdot+\mathrm{e}^{-} & k_{\mathrm{S}}{ }^{\prime} \\
\mathrm{S}^{*}+\mathrm{B} \rightarrow \mathrm{S}+\mathrm{B}^{+} \cdot+\mathrm{e}^{-} & k_{\mathrm{B}} \\
\mathrm{B}^{+} \cdot \mathrm{B} \rightarrow \dot{\mathrm{B}}-\mathrm{B}^{+} & k_{2}{ }^{\prime} \\
\dot{\mathrm{B}}-\mathrm{B}^{+}+\mathrm{B} \rightarrow \mathrm{B}-\mathrm{B}-\mathrm{B}^{+} & \\
\ldots \ldots \ldots \ldots & \ldots \ldots . \\
\dot{\mathrm{B}}-(\mathrm{B})_{n}-\mathrm{B}^{+}+\mathrm{e}^{-} \ldots \dot{\mathrm{B}}-(\mathrm{B})_{n}-\dot{\mathrm{B}}
\end{array}
$$

The difference between Models I and II is only in the mechanism for the butadiene cation radical formation. The initiation of polymerization by the cation radical (3) and propagation by a cationic mechanism (4) and the termination of propagating allyl-type cation with an electron (5) are the same in both Models. Cationic propagation cannot be traced by as ESR spectrometer. In radiation-induced polymerization the termination rate constant is much larger than the propagation rate constant. ${ }^{5}$ The relation of $\left[\mathbf{S}^{+} \cdot\right]+\left[\mathrm{B}^{+} \cdot\right]+[\mathrm{B}-] / 2=\left[\mathbf{S}^{+} \cdot\right]_{0}$ indicates that the termination rate constant is much larger than $k_{2}{ }^{\prime}$. Thus, the kinetics based on each Model are expressed as follows:

\section{Model I}

$$
\frac{\mathrm{d}\left[\mathrm{S}^{+} \cdot\right]}{\mathrm{d} t}=K-k_{1}{ }^{\prime}\left[\mathrm{S}^{+} \cdot\right][\mathrm{B}]
$$


ESR Studies on Primary Processes in Radiation-Induced Reactions

$$
\begin{aligned}
& \frac{\mathrm{d}\left[\mathrm{B}^{+} \cdot\right]}{\mathrm{d} t}=k_{1}{ }^{\prime}\left[\mathrm{S}^{+} \cdot\right][\mathrm{B}]-k_{2}{ }^{\prime}\left[\mathrm{B}^{+} \cdot\right][\mathrm{B}] \\
& \frac{\mathrm{d}[\dot{\mathrm{B}}-]}{\mathrm{d} t}=k_{2}{ }^{\prime}\left[\mathrm{B}^{+} \cdot\right][\mathrm{B}]
\end{aligned}
$$

Model II

$$
\begin{aligned}
& \frac{\mathrm{d}\left[\mathrm{S}^{*}\right]}{\mathrm{d} t}=K-k_{\mathrm{S}}{ }^{\prime}\left[\mathrm{S}^{*}\right]-k_{\mathrm{B}}{ }^{\prime}\left[\mathrm{S}^{*}\right][\mathrm{B}] \\
& \left.\frac{\mathrm{d}\left[\mathrm{S}^{+} \cdot\right]}{\mathrm{d} t}=k_{\mathrm{S}}{ }^{\prime} \mathrm{S}^{*}\right] \\
& \frac{\mathrm{d}\left[\mathrm{B}^{+} \cdot\right]}{\mathrm{d} t}=k_{\mathrm{S}}{ }^{\prime}\left[\mathrm{S}^{*}\right][\mathrm{B}]-k_{2}{ }^{\prime}\left[\mathrm{B}^{+} \cdot\right][\mathrm{B}] \\
& \frac{\mathrm{d}[\dot{\mathrm{B}}-]}{\mathrm{d} t}=k_{2}{ }^{\prime}\left[\mathrm{B}^{+} \cdot\right][\mathrm{B}]
\end{aligned}
$$

By assuming that the consumption of butadiene is small during irradiation, $(\mathbf{B})$ is taken to be a constant $B$, then the differential equations are solved. The yield of each species is expressed as follows:

\section{Model I}

$$
\begin{aligned}
& {\left[\mathbf{S}^{+} \cdot\right]=\frac{K}{k_{1}{ }^{\prime} B}\left(1-\exp \left(-k_{1}{ }^{\prime} B t\right)\right)} \\
& {\left[\mathrm{B}^{+} \cdot\right]=\frac{K}{B}\left(\frac{1}{k_{2}{ }^{\prime}}-\frac{1}{k_{2}{ }^{\prime}-k_{1}{ }^{\prime}} \exp \left(-k_{1}{ }^{\prime} B t\right)\right.} \\
& \left.+\left(\frac{1}{k_{2}^{\prime}-k_{1}^{\prime}}-\frac{1}{k_{2}^{\prime}}\right) \exp \left(-k_{2}^{\prime} B t\right)\right) \\
& {[\dot{\mathrm{B}}-]=\frac{K}{B}\left(B t+\left(\frac{1}{k_{1}{ }^{\prime}}+\frac{1}{k_{2}{ }^{\prime}-k_{1}{ }^{\prime}}\right) \exp \left(-k_{1}{ }^{\prime} B t\right)\right.} \\
& -\left(\frac{1}{{k_{2}{ }^{\prime}-k_{1}{ }^{\prime}}^{\prime}}-\frac{1}{k_{2}{ }^{\prime}}\right) \exp \left(-k_{2}{ }^{\prime} B t\right) \\
& \left.-\frac{1}{k_{1}{ }^{\prime}}-\frac{1}{k_{2}{ }^{\prime}}\right)
\end{aligned}
$$

Model II

$$
\begin{aligned}
& {\left[\mathrm{S}^{*}\right]=\frac{K}{k_{\mathrm{S}}{ }^{\prime}+k_{\mathrm{B}}{ }^{\prime}}\left(1-\exp \left(-\left(k_{\mathrm{S}}{ }^{\prime}+k_{\mathrm{B}}{ }^{\prime} B\right) t\right)\right.} \\
& {\left[\mathrm{S}^{+} \cdot\right]=\frac{K k_{\mathrm{S}}{ }^{\prime}}{k_{\mathrm{S}}{ }^{\prime}+k_{\mathrm{B}}{ }^{\prime} B}\left(t+\frac{\exp \left(-\left(k_{\mathrm{S}}{ }^{\prime}+k_{\mathrm{B}}{ }^{\prime} B\right) t\right)}{k_{\mathrm{S}}{ }^{\prime}+k_{\mathrm{B}}{ }^{\prime}}\right.} \\
& \left.-\frac{1}{k_{\mathrm{S}}{ }^{\prime}+k_{\mathrm{B}}{ }^{\prime} B}\right) \\
& {\left[\mathrm{B}^{+} \cdot\right]=\frac{K k_{\mathrm{B}}{ }^{\prime} B}{k_{\mathrm{S}}{ }^{\prime}+k_{\mathrm{B}}{ }^{\prime} B}\left(\frac{1}{k_{2}{ }^{\prime} B}+\frac{\exp \left(-\left(k_{\mathrm{S}}{ }^{\prime}+k_{\mathrm{B}}{ }^{\prime} B\right) t\right)}{k_{\mathrm{S}}{ }^{\prime}+k_{\mathrm{B}}{ }^{\prime} B-k_{2}{ }^{\prime} B}\right.} \\
& -\left(\frac{1}{k_{2}{ }^{\prime} B}+\frac{1}{k_{\mathrm{S}}{ }^{\prime}+k_{\mathrm{B}}{ }^{\prime} B-k_{2}{ }^{\prime} B}\right) \\
& \left.\times \exp \left(-k_{2}{ }^{\prime} B t\right)\right)
\end{aligned}
$$

$$
\begin{aligned}
& {[\dot{\mathrm{B}}-]=\frac{K k_{\mathrm{B}}{ }^{\prime} k_{2}{ }^{\prime} B^{2}}{{k_{\mathrm{S}}}+k_{\mathrm{B}}{ }^{\prime} B}\left(\frac{t}{k_{2}{ }^{\prime} B}\right.} \\
& +\frac{\left.1-\exp \left(k_{\mathrm{S}}{ }^{\prime}+k_{\mathrm{B}}{ }^{\prime} B\right) t\right)}{\left(k_{\mathrm{S}}{ }^{\prime}+k_{\mathrm{B}}{ }^{\prime} B-k_{\mathrm{2}}{ }^{\prime} B\right)\left(k_{\mathrm{S}}{ }^{\prime}+k_{\mathrm{B}}{ }^{\prime}\right)} \\
& -\frac{1}{k_{2}{ }^{\prime} B}\left(\frac{1}{k_{2}{ }^{\prime} B}\right. \\
& \left.\left.+\frac{1}{{k_{\mathrm{S}}}{ }^{\prime}+{k_{\mathrm{B}}}^{\prime} B-k_{2}{ }^{\prime} B}\right)\left(1-\exp \left(-k_{2}{ }^{\prime} B t\right)\right)\right)
\end{aligned}
$$

The rate constants for both Models I and II are estimated from $\left[\mathbf{S}^{+} \cdot\right],\left[\mathbf{B}^{+} \cdot\right]$ and $[\dot{\mathbf{B}}-]$ in Figure 3 by the best fit procedure using a computer program. The theoretical curves from the estimated rate constants are shown in Figure 3 with a solid line for Model I and a dotted line for Model II. The values estimated are $k_{1}{ }^{\prime}=$ $5.0 \times 10 \mathrm{~g} / \mathrm{mol} \mathrm{s}$ and $k_{2}{ }^{\prime}=3.5 \times 10 \mathrm{~g} / \mathrm{mol} \mathrm{s}$ for Model I, and $k_{\mathrm{S}}{ }^{\prime}=2.0 \times 10^{3} \mathrm{~s}^{-1}, k_{\mathrm{B}}{ }^{\prime}=1.3 \times 10^{8}$ $\mathrm{g} / \mathrm{mol} \mathrm{s}$ and $k_{2}{ }^{\prime}=3.0 \times 10 \mathrm{~g} / \mathrm{mol} \mathrm{s}$ for Model II. Equations 21 and 22 become equal to eq 17 and 18 respectively, when the butadiene concentration increases. The theoretical curves for both Models I and II lie close to the experimental points as shown in Figure 3. This good fit gives strong evidence for the reaction mechanisms of butadiene on silica gel during irradiation: The butadiene cation radical initiates polymerization to form the radical RI on the other end of a propagating chain end. The propagation is reasonably suggested to proceed by allyl-type cation. The termination is the electron-capture process of the propagating allyl-type cation. The mechanism of the butadiene cation radical formation by the interaction with silica gel is expected to be determined from the comparison of the theoretical curves with the experimental points at low butadiene contents. In the present study it is difficult to determine a mechanism, because both models fit as well to the limited number of experimental points. However, it is clear that butadiene acts as an efficient sink for holes in the silica gel. The $G_{i}$-value for the formation of the hole is estimated to be 0.47 .

The rate constant of cationic propagation cannot be estimated by means of ESR. In this adsorbed system the butadiene content on the silica gel is expressed as moles of butadiene per 
gram of silica gel (mol/g). If the apparent density of silica gel is assumed to be about 0.3 , the apparent rate constant of reaction (3) is estimated to be about $1.0 \times 10^{-1} \mathrm{M}^{-1} \mathrm{~s}^{-1}$ from $k_{2}{ }^{\prime}$. This value is for the first step of polymerization, but not for the propagation. The rate constant for the propagation in radiation-induced ionic polymerization in liquid phase has been reported ${ }^{6}$ to be $10^{5}-10^{8} \mathrm{M}^{-1} \mathrm{~s}^{-1}$.

The similar behavior between the hole in silica gel at $g=2.0070$ and the electrons at $g=2.0030$ in the absence of butadiene (Figure 4 and Table I) and the existence of the electrons at $g=2.0008$ even at room temperature imply that the termination reaction is the recombination between propagating allyl-type cations, and the electrons at $g=2.0030$.

Acknowledgment. Support of this work partly by The Swedish Board for Technical Development and The Swedish Atomic Research Council is appreciated. The authors are grateful to The Swedish Research Councils' Laboratory for its excellent facilities and hospitality and to Professor P.O. Kinell and Dr. A. Lund for their encouragement. The authors thank Professor H. Yamaoka at Research Reactor Institute for his encouragement.

\section{REFERENCES}

1. T. Shiga, A. Lund, and P. O. Kinell, Int. J. Radiat. Phys. Chem., 3, 131 (1971).

2. T. Shiga, A. Lund, and P. O. Kinell, Acta Chem. Scand., 25, 1508 (1971).

3. Program ASESK written by T. Vanngard (1961); revised and extended by T. Shiga and A. Lund.

4. P. O. Kinell, T. Komatsu, A. Lund, T. Shiga, and A. Shimizu, Acta Chem. Scand., 24, 3265 (1970).

5. F. Williams, Ka. Hayashi, K. Ueno, K. Hayashi, and S. Okamura, Trans. Faraday Soc., 63, 1501 (1967).

6. F. Williams in "Fundamental Processes in Radiation Chemistry", P. Ausloos, Ed., John Wiley \& Sons, Inc., New York, N.Y., 1968, p. 598. 